\title{
Observation of Velocity-Tuned Multiphoton “Doppleron” Resonances in Laser-Cooled Atoms
}

\author{
J. J. Tollett, J. Chen, J. G. Story, N. W. M. Ritchie, C. C. Bradley, and Randall G. Hulet \\ Physics Department and Rice Quantum Institute, Rice University, Houston, Texas 77251
}

(Received 15 March 1990)

\begin{abstract}
An atomic beam of $\mathrm{Li}$ was transversely cooled using an intense standing-wave radiation field. A dramatic change in the transverse velocity distribution was observed. Structure in the resulting velocity distribution was found to be due to velocity-tuned multiphoton "Doppleron" resonances. The force due to seven-photon resonances is clearly resolved in the data. The data are in good agreement with theoretical predictions.

PACS numbers: $32.80 . \mathrm{Pj}$
\end{abstract}

The forces experienced by an atom moving in an intense standing-wave radiation field are due to several fundamentally different physical mechanisms depending on the velocity of the atom and the intensity of the field. At high intensities, multiphoton effects become important. The force due to multiphoton transitions can be very large and may be of considerable practical interest for the efficient manipulation of atomic motion. These high-intensity effects have not yet been fully investigated. In this paper, we report the observation of a dramatic redistribution of the velocities of atoms in a beam due to multiphoton processes in an intense standing wave.

At sufficiently low intensity, the force on the atom is the sum of the forces from each individual travelingwave component of the standing wave. ${ }^{\prime}$ If the radiation frequency is tuned near, but below, an atomic transition frequency, the atom will experience a net cooling force. This is the "usual" laser cooling force or, the so-called Doppler force. However, at high intensity, the force is fundamentally altered. Stimulated processes involving both traveling waves become important. Figure 1 shows the results of a calculation of the velocity-dependent force on an atom averaged over one optical wavelength of travel, as a function of the atom's velocity. This calculation employs a continued-fraction method to obtain the steady-state solution of the exact density-matrix equations. ${ }^{2}$ The force depends on the on-resonance Rabi frequency $\Omega$, where $\hbar \Omega$ is the energy of interaction between the atom and the field at an antinode of the standing wave, and on the detuning from resonance $\Delta \equiv \omega$ $-\omega_{0}$, where $\omega$ is the radiation frequency and $\omega_{0}$ is a resonance frequency of the atom. The parameters corresponding to the results displayed in Fig. 1 are $\Omega=60 \gamma$ and $\Delta=30 \gamma$, where $\gamma$ is the spontaneous decay rate of the excited state. The force is expressed in units of the maximum Doppler force $\hbar k \gamma / 2$, where $\hbar k$ is the photon momentum, and the velocity is in units of $\gamma / k$. The average velocity-dependent force on a slowly moving atom is opposite in sign to that of the Doppler force. This force, known as the velocity-dependent "dipole" or "gradient" force, depends on the spatial gradient of the electric-field strength and is now well understood. ${ }^{3}$

At high intensity, resonances appear in the average force at certain velocities, as shown in Fig. 1. These velocity-tuned multiphoton resonances have been designated "Doppleron" resonances. ${ }^{4}$ The following simple model provides physical insight into these resonances. The upper part of Fig. 2(a) depicts an atom moving with velocity $v$ in a standing wave of frequency $\omega$ in the laboratory frame. In the frame of the atom, shown in the lower part of Fig. 2(a), the frequencies of the two traveling waves are Doppler shifted, so that the atom experiences counterpropagating traveling waves of different frequency, $\omega^{ \pm}=\omega \pm k v$. Figure 2(b) illustrates the Doppleron resonances for the case that $\Delta<0$. The onephoton resonance condition will be satisfied if $\omega_{1}^{+}$equals the frequency difference between the atomic states. This occurs for a velocity which satisfies $|\Delta|=k v_{1}$. For cer-

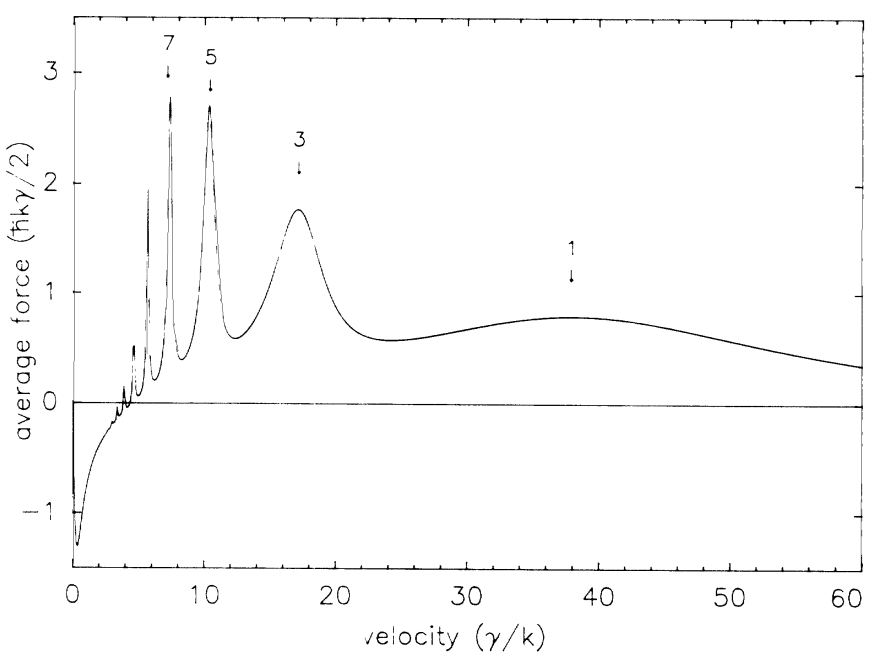

FIG. 1. Calculational results of the average force on an atom in a standing wave as a function of the velocity of the atom. The force is given in units of the maximum Doppler force. The value of the peak Rabi frequency is $\Omega=60 \gamma$, and the detuning of the standing-wave frequency from resonance is $\Delta=30 \gamma$, where $\gamma$ is the radiative decay lifetime. The average force is a cooling force for slowly moving atoms, where the dipole force is dominant. At higher velocity, the force changes sign and velocity-resonant Doppleron structure appears. The one-photon through seven-photon Doppleron resonances are indicated. 


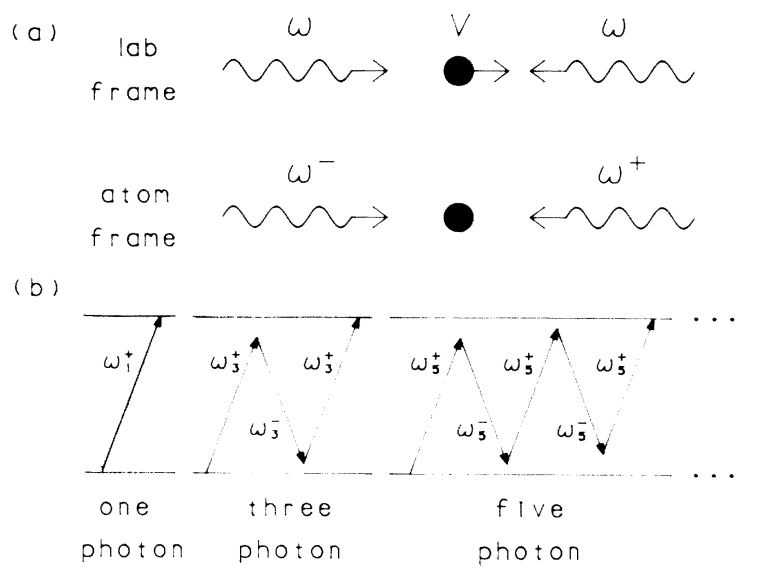

FIG. 2. (a) An atom travels at velocity $v$ in the laboratory frame in the presence of counterpropagating, equal-frequency traveling waves. In transforming to the frame of the atom, the frequencies of the traveling waves acquire equal, but opposite Doppler shifts: $\omega_{ \pm}=\omega \pm k v$. (b) Assume that $\omega$ is below the resonant frequency of the atom by an amount $\Delta$. A one-photon transition will be resonant for $|\Delta|=k v$. Similarly, multiphoton stimulated Raman transitions involving the absorption and stimulated emission of photons from both traveling waves will be resonant for certain velocities. The resonance conditions are $|\Delta|=n k v$, where $n$ is an odd integer. These are the velocitytuned Doppleron resonances.

tain velocities $v<v_{1}$, multiphoton resonance conditions will be satisfied. A three-photon stimulated Raman process can occur if the atom absorbs an $\omega^{+}$photon, is stimulated to emit an $\omega^{-}$photon, and finally absorbs another $\omega^{+}$photon. This process is resonant for $|\Delta|$ $=3 k v_{3}$. Similarly, higher-order processes involving an odd number of stimulated events can occur at velocities satisfying the condition $|\Delta|=n k v$, where $n$ is an odd integer. The term Doppleron refers to the quanta of energy corresponding to the Doppler shift, $\hbar k v$.

Many of the features of the force spectrum of Fig. 1 can be understood with reference to this model. The first few Doppleron resonances are indicated in Fig. 1 by arrows. The force due to the one-photon process, the Doppler force, is saturated at a force of 1 in these units, as expected. This feature occurs near $v=\Delta / k$ and is significantly power broadened by the intense field. The peaks corresponding to the higher-order processes are progressively narrower since the rate for these becomes correspondingly weaker. The force due to the $n$ th-order Doppleron process can be $n$ times larger than the saturated Doppler force. However, the force due to the highest-order processes are weaker because of their relative inefficiency. The resonant velocities predicted by this simple model differ somewhat from those predicted by the numerical exact theory. This difference is due to the significant perturbation of the atomic energy levels by the intense standing-wave field.

The existence of velocity-tuned multiphoton atomic resonances was first predicted by Haroche and Hart-

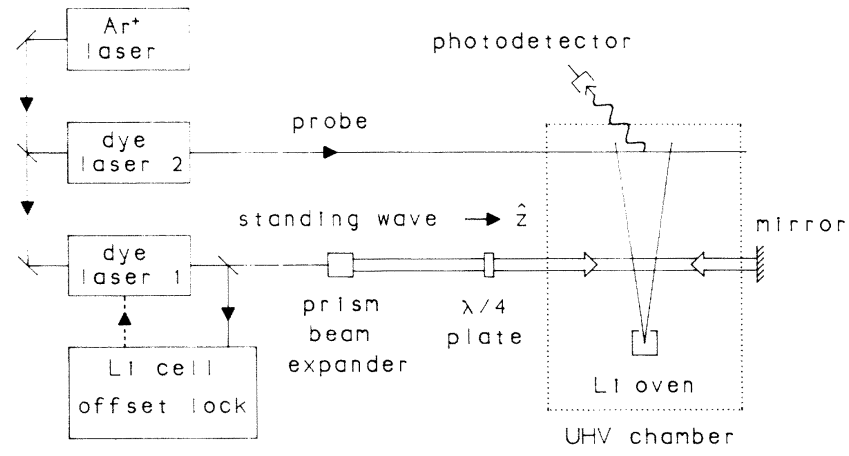

FIG. 3. Schematic diagram of the experimental apparatus. A thermal beam of $\mathrm{Li}$ atoms is intersected by an intense standing wave, which is formed by retroreflecting a laser beam from a continuous-wave, ring dye laser. The frequency of the standing wave is locked near the $2 S_{1 / 2}, F=2 \leftrightarrow 2 P_{3 / 2}, F=3$ transition frequency. The transverse velocity distribution downstream of the standing wave is probed by detecting the fluorescence induced by a second dye laser which is scanned through the transition frequency.

mann in the context of saturated-absorption spectrosco$\mathrm{py}^{5}$ and the predicted line shapes were subsequently observed. ${ }^{6}$ More recently, Dopplerons have been employed in the cooling of the longitudinal velocity of atoms in a beam. ${ }^{7}$ However, the force due to individual Doppleron resonances has not been previously resolved.

The experimental setup is shown schematically in Fig. 3. A $\mathrm{Li}$ atomic beam is intersected at right angles by a standing wave formed by retroreflecting the output beam of a single-frequency, continuous-wave ring dye laser (dye laser 1). The standing-wave axis is taken to be the $z$ axis. In order to increase the interaction time of the atoms in the standing wave, the laser beam is expanded along the atomic beam axis (horizontal) using four prisms, producing a Gaussian beam waist of $\sim 9.3 \mathrm{~mm}$ in the horizontal direction while the waist in the vertical direction is $\sim 1.1 \mathrm{~mm}$. The laser frequency is tuned near the $2 S_{1 / 2}, F=2 \leftrightarrow 2 P_{3 / 2}, F=3$ resonance frequency of the ${ }^{7} \mathrm{Li}$ atom (671-nm wavelength). An effective two-state cycling system is formed using a quarter-wave plate to circularly polarize the radiation so that atoms in the $F=2, m_{F}=2$ ground state can be excited only to the $F=3, m_{F}=3$ excited state. Atoms not initially in the $F=2, m_{F}=2$ ground state will be optically pumped into it, from where they can participate in the cooling process, or they decay into the $F=1$ ground-state hyperfine level (803-M Hz hyperfine splitting) and are effectively removed from the cooling and detection processes. The laser power was $760 \mathrm{~mW}$, giving a peak intensity in the standing wave of $19 \mathrm{~W} / \mathrm{cm}^{2}$. The saturation intensity of the $F=2, m_{F}=2 \leftrightarrow F=3, m_{F}=3$ transition using circularly polarized light is $5.1 \mathrm{~mW} / \mathrm{cm}^{2}$, yielding a peak onresonance Rabi frequency of $\Omega \approx 60 \gamma$. The interaction of the atoms with the laser defines the quantization axis, since this interaction is larger than that due to any other 
field, including the Earth's magnetic field. The laser beam was apertured in the horizontal direction to a 6 $\mathrm{mm}$ width so that the variation in the Rabi frequency was ideally no more than $10 \%$ as the atoms traversed the standing wave. We estimate that our uncertainty in the Rabi frequency is $8 \%$, mainly due to the measurement of the beam waists. The temperature of the $\mathrm{Li}$ oven was approximately $510^{\circ} \mathrm{C}$, which gives the most probable longitudinal velocity for an atom in the beam of $-1.6 \times 10^{5} \mathrm{~cm} / \mathrm{s}$. An atom with this velocity will spend $3.6 \mu \mathrm{s}$ in the beam, corresponding to $\sim 132$ natural lifetimes of the upper state. This is sufficiently long to ensure that the atoms experience the average force, as shown in Fig. 1, and to ensure that the velocity distribution will be substantially affected.

Dye laser 1 is frequency locked to the $2 S_{1 / 2}, F=2$ $\leftrightarrow 2 P_{3 / 2}$ saturated-absorption line of ${ }^{7} \mathrm{Li}$ in a separate vapor cell. Two acousto-optic modulators are employed to provide both the frequency modulation required for locking and a selected frequency offset of up to 300 $\mathrm{MHz}$ from resonance. Our uncertainty in $\Delta$ is approximately $10 \mathrm{MHz}$ due to the possibility of locking to different hyperfine levels of the excited state. Frequency deviations about the lock point are less than $2 \mathrm{MHz}$.

The velocity distribution of the atoms along the $z$ axis is determined by a weak probe laser (dye laser 2) parallel to the standing wave. The probe-laser frequency is tuned across the $2 S_{1 / 2}, F=2 \leftrightarrow 2 P_{3 / 2}$ transition while a portion of the emitted fluorescence is detected with a silicon photodiode. The Doppler width of the laser-induced fluorescence spectrum of the unperturbed atoms (i.e., standing wave blocked) is approximately $270 \mathrm{MHz}$. This width is determined mostly by the acceptance angle of the photodetector and partially by an aperture in the atomic beam. The short-term frequency fluctuations of the probe laser are much less than the $5.8-\mathrm{MHz}$ natural linewidth of the transition.

Figure 4(a) is a plot of data for which the standingwave parameters are $\Omega=60 \gamma$ and $\Delta=+30 \gamma$ (as in Fig. 1). The horizontal axis of the plot corresponds to the probe-frequency detuning $v$, which is related to the $z$ component of velocity $v_{z}$ by $v=k v_{z} / 2 \pi$. Atoms which are resonant with the probe at zero detuning correspond to atoms with $v_{z}=0$. We identify the structure in the velocity distribution as due to Doppleron resonances. Since $\Delta$ is positive, the Doppleron resonances should give rise to a heating force. The dips in the velocity distribution are due to the depletion of the heated atoms at the velocities corresponding to these resonances. The heated atoms accumulate at larger velocity, where the force is relatively weak, and form the peaks adjacent to the dips. The arrows in the figure indicate the positions of the one-, three-, five-, and seven-photon Doppleron resonances. The resolution of the probe laser is limited by the natural width of the transition, which corresponds to a velocity of $390 \mathrm{~cm} / \mathrm{s}$. This resolution is insufficient to resolve beyond the seven-photon Doppleron resonance.
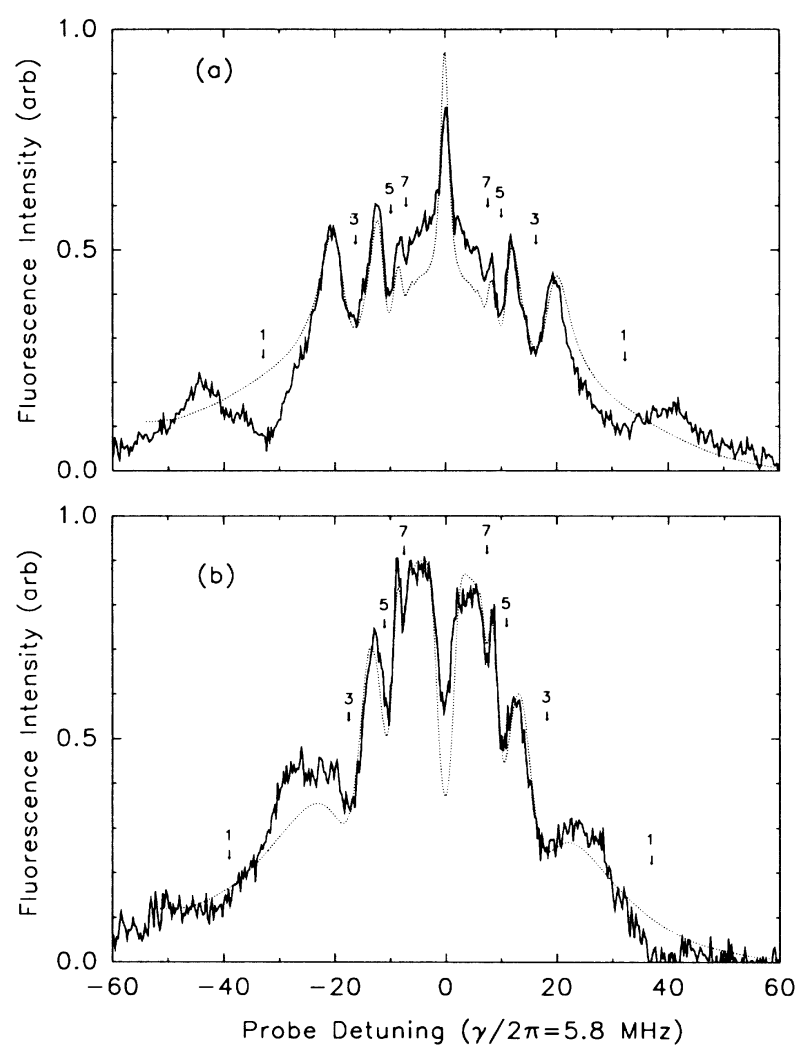

FIG. 4. (a) Laser-induced fluorescence spectrum of the transverse velocity distribution of the atoms after interacting with the standing wave. The horizontal axis is the detuning of the probe frequency from resonance, measured in natural linewidths (the natural linewidth corresponds to a velocity of 390 $\mathrm{cm} / \mathrm{s})$. The standing-wave detuning is $\Delta=+30 \gamma(175 \mathrm{MHz})$ and the Rabi frequency is $\Omega=60 \gamma$ (peak radiation intensity of $19 \mathrm{~W} / \mathrm{cm}^{2}$ ). The depleted regions of the velocity spectrum coincide with the velocities at which the Doppleron resonances occur (indicated by arrows) and the force is relatively large. These atoms are heated and accumulate at higher velocity where the force is relatively weak. The narrow central peak, at very low velocity, is a result of the velocity-dependent dipoleforce cooling. The slight asymmetry in the data is the result of a slight asymmetry in the original, unperturbed velocity distribution relative to the $z$ axis. The dotted line is a fit to the data. (b) Same as (a), except that $\Delta=-30 \gamma$, causing the sign of the force to be reversed. The effect of the Doppleron resonances is to cool the atoms. The velocity-dependent dipole force is heating for low velocity, producing the central dip.

The velocity-dependent dipole force, which is a cooling force for small velocities, is responsible for the narrow central peak.

Also shown in Fig. 4(a) is a fit resulting from numerical integration of the atoms' motion through the standing wave. The inputs to this fit are the unperturbed velocity distribution, obtained by probing the atoms with no standing wave present, and the results of the exact force calculation displayed in Fig. 1. The longitudinal velocity distribution, which causes a spread in interaction 
time with the standing wave, and the velocity resolution due to the natural linewidth of the atomic transition are included in the fit. The only fitting parameters are the overall normalization and the position of zero velocity. The frequency scale was adjusted slightly to improve the fit, but was consistent with our uncertainty in the measured probe scan width. Overall, the calculation reproduces the positions and magnitude of the Doppleron features very precisely. However, the calculation does not reproduce the magnitude of the one-photon feature observed in our data. We believe that this feature is due to the atoms traversing a portion of the standing wave which is much less intense than the center of the beam. The lower intensity causes the one-photon resonance to be narrower, and therefore, more pronounced.

Figure 4(b) is similar to Fig. 4(a) except that the detuning is negative, $\Delta=-30 \gamma$. The dips in the velocity distribution are due to the Doppleron resonances, as before. However, the Doppleron resonances give rise to a cooling force for this detuning and the atoms are slowed to lower force regions where they collect. The velocitydependent dipole force for small velocity is heating, thereby producing a dip at zero velocity, rather than a peak as in Fig. 4(a). The effect of the higher-order resonances is more pronounced for negative detuning than for positive, because cooling narrows the velocity distribution, producing sharper features. We further confirmed our identification of the effect of the Doppleron resonances by obtaining data for several values of $\Delta$. Again, we find that the data agree with the theoretical fits.

Our experiment is similar to previous experiments which have investigated the force on an atom due to an intense transverse standing wave. ${ }^{8,9}$ However, these experiments were concerned mainly with the dipole force which dominates for very low velocity. In these experiments, the atoms' transverse spatial positions were detected with a hot-wire surface-ionization method. With this method, the resolution of large transverse velocity is reduced due to the longitudinal velocity distribution in the beam, thereby rendering the Doppleron resonances unobservable. A probe laser, as used in our experiment, directly measures the transverse velocity distri- bution.

In summary, we have observed structure in the transverse velocity distribution of atoms which have traversed an intense standing wave. We confirmed that this structure is produced by the force due to multiphoton Doppleron resonances by comparing data for several values of the radiation frequency detuning to a numerically exact calculation. The effect of multiphoton resonances up to seventh order are clearly visible in the velocity spectrum.

We note that subsequent to the preparation of this manuscript, we received a preprint which reports the observation of the effect of Doppleron resonances on the longitudinal velocity distribution of atoms moving in a standing wave. ${ }^{10}$

We are grateful to J. C. Bergquist for help with the frequency-offset locking technique. This material is based in part upon work supported by the Texas Advanced Technology Program, a National Institute of Standards and Technology Precision Measurement Grant, the Welch Foundation, and the National Science Foundation. R.G.H. is an Alfred P. Sloan Research Fellow.

${ }^{1}$ D. J. Wineland and W. M. Itano, Phys. Rev. A 20, 1521 (1979).

${ }^{2}$ V. G. Minogin and O. T. Serimaa, Opt. Commun. 30, 373 (1979)

${ }^{3}$ J. Dalibard and C. Cohen-Tannoudji, J. Opt. Soc. Am. B 2, 1707 (1985).

${ }^{4}$ E. Kyrölä and S. Stenholm, Opt. Commun. 22, 123 (1977).

${ }^{5}$ S. Haroche and F. Hartmann, Phys. Rev. A 6, 1280 (1972).

${ }^{6}$ S. M. Freund, M. Römheld, and T. Oka, Phys. Rev. Lett. 35, 1497 (1975).

${ }^{7}$ M. Prentiss and A. Cable, Phys. Rev. Lett. 62, 1354 (1989).

${ }^{8}$ A. Aspect, J. Dalibard, A. Heidmann, C. Salomon, and C. Cohen-Tannoudji, Phys. Rev. Lett. 57, 1688 (1986).

${ }^{9}$ C. E. Tanner, B. P. Masterson, and C. E. Weiman, Opt. Lett. 13, 357 (1988).

${ }^{10}$ N. P. Bigelow and M. G. Prentiss, preceding Letter, Phys. Rev. Lett. 65, 555 (1990). 